CONFORMAL GEOMETRY AND DYNAMICS

An Electronic Journal of the American Mathematical Society

Volume 14, Pages 256-268 (October 13, 2010)

S 1088-4173(2010)00214-6

\title{
ON DYNAMICAL TEICHMÜLLER SPACES
}

\author{
CARLOS CABRERA AND PETER MAKIENKO
}

\begin{abstract}
Following ideas from a preprint of the second author (see Automorphisms of a rational function with disconnected Julia set, Orsay, Preprint, 03 1992), we investigate relations of dynamical Teichmüller spaces with dynamical objects. We also establish some connections with the theory of deformations of inverse limits and laminations in holomorphic dynamics (see J. Diff. Geom. 47 (1997), 17-94).
\end{abstract}

\section{INTRODUCTION}

Sullivan introduced the study of a dynamical Teichmüller space, which we denote by $T_{1}(R)$, associated to a rational function $R$. The space of orbits of $T_{1}(R)$, under the action of an associated modular group $\operatorname{Mod}_{1}(R)$, coincides with the space $Q C(R)$ of quasiconformal deformations of $R$. We modify Sullivan's definition to get another Teichmüller space $T_{2}(R)$, with its corresponding modular group $\operatorname{Mod}_{2}(R)$. In this situation, the $J$-stability component is the space of orbits of $T_{2}(R)$, under the action of $\operatorname{Mod}_{2}(R)$. When $R$ is hyperbolic, the $J$-stability component is the hyperbolic component of $R$.

There are natural inclusions of the space $T_{1}(R)$ into $T_{2}(R)$, and from the group $\operatorname{Mod}_{1}(R)$ into $\operatorname{Mod}_{2}(R)$. We find that properties of these inclusions are related to the dynamics of $R$. With this in hand, we can establish relations between the dynamics of $R$ and topological properties of $T_{2}(R)$.

When the Julia set of $R$ is totally disconnected, the space $T_{2}(R)$ has a laminated structure. In this way, we also realize $T_{2}(R)$ as the space of deformations of the natural extension of $R$. The structure of the paper is as follows.

In Section 2, we recall basic definitions and facts of the classical Teichmüller space $T_{1}(R)$.

In Section 3, we introduce $T_{2}(R)$ and show that, as in the case of $T_{1}(R)$, it is a complete metric space. In Theorem 3.5. we establish characterizations for the path connectivity of $T_{2}(R)$. Using this, we prove Theorem 3.7 stating that, when $R$ is a polynomial, the connectivity of the Julia set $J(R)$ is equivalent to the path connectivity of $T_{2}(R)$.

In Section 4, we restrict to the case where $J(R)$ is homeomorphic to a Cantor set. In this case, $T_{2}(R)$ is a trivial product of $T_{1}(R)$ times a totally disconnected space. We finish the section giving a characterization of the property that $J(R)$ is homeomorphic to a Cantor set in terms of properties of $T_{2}(R)$.

Received by the editors November 30, 2009.

2010 Mathematics Subject Classification. Primary 37F30, 37F10; Secondary 37F50.

Key words and phrases. Holomorphic dynamics, Teichmüller theory.

This work was partially supported by PAPIIT project IN 100409.

(C) 2010 American Mathematical Society Reverts to public domain 28 years from publication 
Finally, in Section 5, we construct a realization of $T_{2}(R)$ as the space of deformations of the natural extension of $R$.

\section{The TeIChmüller SPACE $T_{1}(R)$}

Given a rational map $R$, let us define the space $T_{1}(R)=T\left(S_{R}\right) \times B_{R}$, where $S_{R}$ is a Riemann surface associated to the Fatou set $F(R), T\left(S_{R}\right)$ denotes the classical Teichmüller space of $S_{R}$, and $B_{R}$ is the space of invariant Beltrami differentials, defined on the Julia set $J(R)$, which are compatible with the dynamics of $R$. That is, $B_{R}$ is the space of measurable $(1,-1)$ forms $\mu$ with $L^{\infty}$ norm bounded by 1 , satisfying the conditions that $\mu$ is 0 outside the Julia set $J(R)$ and $R^{*}(\mu)=\mu$. For a more detailed account of the definitions, see 5 and 6 .

An equivalent way to define $T_{1}(R)$ is as the set of isotopy classes of pairs $\left\langle\left[R_{1}\right],[\phi]\right\rangle$ where $\phi$ is a quasiconformal conjugation of $R$ to the rational map $R_{1}$. The first modular group $\operatorname{Mod}_{1}(R)$, is the group of all isotopy classes of quasiconformal homeomorphisms of $\mathbb{C}$ commuting with $R$. The group $\operatorname{Mod}_{1}(R)$ acts on $T_{1}(R)$ with the action given by

$$
[\phi]\langle[g],[\psi]\rangle=\left\langle[g],\left[\psi \circ \phi^{-1}\right]\right\rangle .
$$

A theorem due to Sullivan and McMullen states that $\operatorname{Mod}_{1}(R)$ acts on $T_{1}(R)$ as a group of isometries; for more details, see [5] and [6]. The formula $T_{1}(R) / \operatorname{Mod}_{1}(R)=$ $Q C(R)$, where $Q C(R)$ is the space of quasiconformal deformations of $R$, will play an important role in what follows.

\section{THE SPACE $T_{2}(R)$}

We will define a Teichmüller space that generalizes the formula

$$
T_{1}(R) / \operatorname{Mod}_{1}(R)=Q C(R)
$$

for the $J$-stability component of $R$.

Definition 3.1. Let $\left(X, d_{1}\right)$ and $\left(Y, d_{2}\right)$ be metric spaces, a map $\phi: X \rightarrow Y$ is called $K$-quasiconformal, in Pesin's sense if, for every $x_{0} \in X$,

$$
\limsup _{r \rightarrow 0}\left\{\frac{\sup \left\{\left|\phi\left(x_{0}\right)-\phi\left(x_{1}\right)\right|:\left|x_{0}-x_{1}\right|<r\right\}}{\inf \left\{\left|\phi\left(x_{0}\right)-\phi\left(x_{1}\right)\right|:\left|x_{0}-x_{1}\right|<r\right\}}\right\} \leq K .
$$

Let us recall that two rational maps $R_{1}$ and $R_{2}$ are $J$-equivalent, if there is a homeomorphism $h: J\left(R_{1}\right) \rightarrow J\left(R_{2}\right)$, which is quasiconformal in Pesin's sense and conjugates $R_{1}$ to $R_{2}$.

Given a family of maps $\left\{R_{w}\right\}$ depending holomorphically on a parameter $w \in W$, a map $R_{w_{0}}$ in $\left\{R_{w}\right\}$ is called $J$-stable if there is a neighborhood $V$ of $w_{0}$ such that $R_{w}$ is $J$-equivalent to $R_{w_{0}}$ for all $w \in V$, and the conjugating homeomorphisms depend holomorphically on $w$.

We denote by $Q C_{J}(R)$, the $J$-stability component of a rational map $R$. This is the path connected component of the $J$-equivalence class of $R$ containing $R$. In [4, Mañe, Sad and Sullivan proved that for every holomorphic family of rational maps, the union of the $J$-stability components is open and dense. When $R$ is hyperbolic, an application of the $\lambda$-Lemma, for holomorphic motions around $J(R)$, shows that $Q C_{J}(R)$ coincides with $\operatorname{Hyp}(R)$, the hyperbolic component of $R$. 
Let $R$ be a rational map. We define the space $X_{n}(R)$ as the set of pairs $(h, U)$, where $U$ is an open neighborhood of the Julia set $J(R)$ and $h: U \rightarrow \mathbb{C}$ is a quasiconformal embedding such that

$$
h \circ R \circ h^{-1}=R_{h}
$$

is the restriction of the rational map, with $\operatorname{deg} R \leq \operatorname{deg} R_{h} \leq n$, wherever the conjugacy is well defined.

We say that $\left(h_{1}, U_{1}\right) \sim\left(h_{2}, U_{2}\right)$ in $X_{n}(R)$ if, and only if, there exist open sets $V_{1}$ and $V_{2}$, satisfying $V_{i} \subset U_{1} \cap U_{2}, J(R) \subset V_{i}$, for $i=1,2$, and a Möbius transformation $\gamma: \mathbb{C} \rightarrow \mathbb{C}$ such that the following diagram commutes

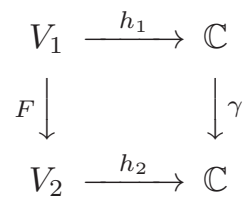

and so that $F$ is a map homotopic to the identity, with a homotopy that commutes with $R$.

With this equivalence relation on $X_{n}(R)$, we can take representatives $(h, U)$ such that, $U$ has nice dynamical properties. For instance, if $R$ is hyperbolic, we can always choose $U$ satisfying $R^{-1}(U) \subset U$.

Following classical Teichmüller theory, the map $\gamma$ would be a holomorphic map. However, the next proposition justifies our definition. The arguments of the proof of this proposition were given to the second named author by A. Eremenko; he also pointed out that these arguments were already present in the work of Fatou. The proposition, and a more general statement, was proved by Buff and Epstein in [1]. For completeness, we include the arguments of the proof here.

Proposition 3.2. Let $R_{1}$ and $R_{2}$ be rational maps, and let $\gamma$ be a conformal map that conjugates $R_{1}$ to $R_{2}$ around a neighborhood of $J\left(R_{1}\right)$. Then $\gamma$ is the restriction of a Möbius transformation.

Proof. Let $U$ be the neighborhood around $J\left(R_{1}\right)$ on which $\gamma$ is defined, and let $x$ be a point in $U$; we define $\gamma_{x}\left(R_{1}(x)\right)=R_{2}(\gamma(x))$ and analytically continue $\gamma$ on $R_{1}(U)$ through arcs starting at $R_{1}(x)$. In this way, we obtain a possibly multivalued extension $\gamma_{x}$ of $\gamma$. By construction, $\gamma_{x}$ also conjugates $R_{1}$ to $R_{2}$. Now let $y \in R_{1}^{-1}\left(R_{1}(x)\right)$. Using the branch induced by $y$, we can define another extension $\gamma_{y}$ of $\gamma$ by putting $\gamma_{y}\left(R_{1}(x)\right)=R_{2}(\gamma(x))$, and analytically continuing $\gamma_{y}$ along paths. Now, $\gamma_{x}$ and $\gamma_{y}$ coincide in $U$; hence, by the Monodromy Theorem $\gamma_{x}=\gamma_{y}$ in all $R_{1}(U)$. Thus the extension of $\gamma$ on $R_{1}(U)$ does not depend on branches and is a well-defined holomorphic map. By induction, we extend $\gamma$ to $\bigcup_{m}^{\infty} R_{1}^{m}(U)$. However, since $U$ contains $J\left(R_{1}\right)$, the set $\bigcup_{m}^{\infty} R_{1}^{m}(U)$ covers the whole Riemann sphere, with the exception of at most two points. Hence, $\gamma$ extends to a unimodal holomorphic function defined on the sphere, so $\gamma$ is a Möbius transformation.

Let $T_{2, n}(R)=X_{n}(R) / \sim$. This definition generalizes the notion of the Teichmüller space for a rational function. The space $X_{n}(R)$ is extremely big. Note that we can change the neighborhood $U$, arbitrarily in the pair $(h, U)$, and still get the same point in $T_{2, n}(R)$; for instance, by taking the restriction of $h$ on a smaller neighborhood. Consider the space $T_{2, n}\left(z^{2}\right)$ with $n \geq 3$, this space contains all maps 
of the form $z^{2}+\lambda z^{3}$ for $\lambda$ small enough. In this paper, we will restrict ourselves to the case where $n=\operatorname{deg}(R)$ and, in this situation, we will omit the subindex $n$.

Two quasiconformal maps $f: U \rightarrow V$ and $g: U^{\prime} \rightarrow V^{\prime}$, defined on neighborhoods of $J(R)$, are equivalent $f \sim g$, if there exist $W \subset U \cap U^{\prime}$ on which $f$ and $g$ are homotopic, with a homotopy that commutes with $R$. We can define a modular group

$$
\operatorname{Mod}_{2}(R)=\{h: U \rightarrow V \text { q.c }: h \text { commutes with } R, J(R) \subset U\} / \sim .
$$

Let $R$ be a hyperbolic rational map. One can check that

$$
\operatorname{Hyp}(R) \cong T_{2}(R) / \operatorname{Mod}_{2}(R) .
$$

Note that the group $\operatorname{Mod}_{2}(R)$ does not depend on $n$. For $n>\operatorname{deg} R$, the quotient $T_{2, n}(R) / \operatorname{Mod}_{2}(R)$ forms a much bigger space containing $H y p(R)$; it also contains other components, coming from higher degrees, arranged on the boundary of $\operatorname{Hyp}(R)$. This construction allows us to consider, as basic points of the Teichmüller space, points that "belong" to the boundary of other $T_{2}\left(R^{\prime}\right)$. For instance, $z^{2}$ "belongs" to the boundary of the space $T_{2}\left(\lambda z^{3}+z^{2}\right)$ for $\lambda$ close to zero, but not zero. In fact, the same is true for $T_{1}\left(\lambda z^{3}+z^{2}\right)$. Nevertheless, the complete picture is yet to be understood.

Now, let us define a third modular group $\operatorname{Mod}_{3}(R)$, as the group of maps $\phi$ : $J(R) \rightarrow J(R)$ which are quasiconformal in Pesin's sense and commute with $R$.

One would be inclined to introduce a third Teichmüller space $T_{3}(R)$. A sensible definition for this space is to consider the set of quasiconformal maps, in the sense of Pesin, defined just in $J(R)$ and commuting with $R$. However, it is not clear how to relate this Teichmüller space with the usual quasiconformal theory. In other words, in general, it is not clear if the natural map from $\operatorname{Mod}_{2}(R)$ to $\operatorname{Mod}_{3}(R)$ is surjective. We can carry on this discussion when the map $R$ is hyperbolic and, more generally, when the Julia set is described as limits of telescopes with bounded geometry. In these cases, every quasiconformal map defined on the Julia set and inducing an isomorphism on telescopes can be extended to a quasiconformal map defined on a neighborhood of $J(R)$. For the definition of telescopes, see 7 .

3.1. The space $T_{2}(R)$ is a complete metric space. Consider the formula,

$$
d([f],[g])=\inf \log K\left(g^{-1} \circ f\right),
$$

where $K$ denotes the distortion, and the infimum is taken over all representatives of the maps $f$ and $g$. This formula defines a pseudodistance on equivalence classes of quasiconformal maps. In particular, it defines a distance on the space $T_{1}(R)$; see [6].

Theorem 3.3. The Teichmüller pseudodistance on $T_{2}(R)$ defines a distance and, with this distance, $T_{2}(R)$ is a complete metric space.

Proof. The map $d$ clearly is positive, reflexive, and satisfies the triangle inequality. Let us check that $d$ is non-degenerate.

Let $\left(\phi_{n}, U_{n}\right)$ be a sequence of representative points in $T_{2}(R)$, such that the distortion $K\left(\phi_{n}\right)$ converges to 1 . Note that the neighborhoods $U_{n}$ may converge to the Julia set in the sense of Hausdorff. Hence, let us check that the maps $\phi_{n}$ are eventually well defined over a neighborhood $U$ of $J(R)$. Then, we show that in $U$, the maps $\phi_{n}$ converge to a holomorphic map $\phi$. This will finish the proof, because if $d([f],[g])=0$, then $f$ and $g$ are related by a holomorphic map. 
First let us assume that $R$ is hyperbolic. Consider a repelling fixed point $x_{0}$ of $R$ in $J(R)$, and a neighborhood $W$ around $x_{0}$. Choose $W$ so that the diameter $\operatorname{diam}(W)$ is less than half the distance of $x_{0}$ to the critical set of $R$. With this choice the map $R$ is injective in $W$. We extend the definition of $\phi_{n}$ to $W$ using the formula $\phi_{n}(R(z))=R\left(\phi_{n}(z)\right)$. The same construction works around all repelling periodic points. Since the map is hyperbolic, this construction extends the definition of $\phi_{n}$ to a neighborhood $U$ of $J(R)$, that only depends on the distance of $J(R)$ to the critical set. The space of quasiconformal maps with bounded distortion is compact, then the maps $\phi_{n}$ converge to a holomorphic map on $W$.

When $R$ is not hyperbolic, the argument is more subtle. Since there are critical points on the boundary and nearby, the diameters of the corresponding neighborhoods converge to zero. However, we still can extend the domains of $\phi_{n}$. To do so, take neighborhoods around the critical values in the Julia set and extend to the critical points using the formula $\phi_{n}(R(z))=R\left(\phi_{n}(z)\right)$.

A slight modification in the argument above also shows that every Cauchy sequence in $T_{2}(R)$ converges; thus, $T_{2}(R)$ is a complete metric space.

3.2. The homomorphisms $\alpha$ and $\beta$. Each class of maps in $\operatorname{Mod}_{1}(R)$ belongs to a class of maps in $\operatorname{Mod}_{2}(R)$, and correspondingly in $\operatorname{Mod}_{3}(R)$. So, we have the following chain of homomorphisms

$$
\operatorname{Mod}_{1}(R) \stackrel{\alpha}{\longrightarrow} \operatorname{Mod}_{2}(R) \stackrel{\beta}{\longrightarrow} \operatorname{Mod}_{3}(R) .
$$

The whole sphere is a neighborhood of the Julia set, hence a class of maps in $T_{1}(R)$ uniquely determines a class of maps in $T_{2}(R)$. This gives a map $H: T_{1}(R) \rightarrow$ $T_{2}(R)$. Let us remark that the map $H$, in general, is not injective nor surjective. However, the properties of the map $H$ are connected with the homomorphism $\alpha$.

Proposition 3.4. For any rational map $R$, we have

$$
H\left(T_{1}(R)\right) \cong T_{1}(R) / \operatorname{ker} \alpha .
$$

Proof. Consider the following commutative diagram:

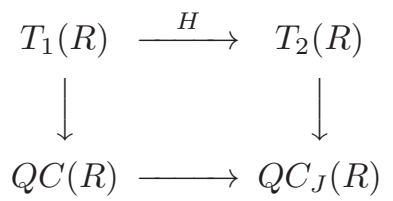

We use the formulae $T_{1}(R) / \operatorname{Mod}_{1}(R)=Q C(R)$ and $T_{2}(R) / \operatorname{Mod}_{2}(R)=Q C_{J}(R)$. Assume that $H\left(\phi_{1}\right)=H\left(\phi_{2}\right)$, then there are neighborhoods $U_{1}, U_{2}, V_{1}, V_{2}$ and a Möbius map $\gamma$ such that the following diagram commutes

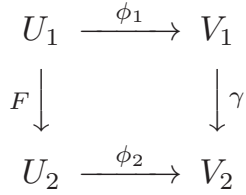

and, the map $F=\phi_{2}^{-1} \circ \gamma \circ \phi_{1}$ is homotopic to $I d$ in $U$, with a homotopy that commutes with dynamics. If $\phi_{1} \neq \phi_{2}$ in $T_{2}(R)$, then the homotopy cannot be extended to a global map in the plane. Since $H\left(\phi_{1}\right)=H\left(\phi_{2}\right)$, the images of $\phi_{1}$ and $\phi_{2}$, under $H$, project to the same element in $Q C_{J}(R)$. By the commutativity of the diagram $\left(^{*}\right), \phi_{1}$ and $\phi_{2}$ project to the same element in $Q C(R)$. Hence, $\phi_{1}$ 
and $\phi_{2}$ are related by a non-trivial element $\psi \in \operatorname{Mod}_{1}(R)$ satisfying $\alpha(\psi)=I d$. So we have $H\left(T_{1}(R)\right) \cong T_{1}(R) / \operatorname{ker} \alpha$.

When $R$ is structurally stable, the map from $Q C(R)$ to $Q C_{J}(R)$, is an embedding with dense image.

Theorem 3.5. Let $R$ be a structurally stable rational map. The following conditions are equivalent:

- The homomorphism $\alpha$ is surjective.

- The set $H\left(T_{1}(R)\right)$ is dense in $T_{2}(R)$.

- The space $T_{2}(R)$ is path connected.

Proof. Assume that the homomorphism $\alpha$ is surjective. Again, we make use of the diagram $\left(^{*}\right)$. Given any $\epsilon>0$ and a point $x \in T_{2}(R)$, there exists $y \in H\left(T_{1}(R)\right)$ and $\phi \in \operatorname{Mod}_{2}(R)$ such that $d(\phi(y), x)<\epsilon$. Since $\alpha$ is surjective, there exists $\psi \in \operatorname{Mod}_{1}(R)$ such that $\alpha(\psi)=\phi$. But this implies that $\phi(y) \in H\left(T_{1}(R)\right)$. Thus the set $H\left(T_{1}(R)\right)$ is dense in $T_{2}(R)$.

Let us assume that $H\left(T_{1}(R)\right)$ is dense in $T_{2}(R)$, take two points $x$ and $y$ in $T_{2}(R)$, then there are two sequences $\left\{x_{n}\right\}$ and $\left\{y_{n}\right\}$ in $H\left(T_{1}(R)\right)$ converging to $x$ and $y$, respectively. Since $T_{1}(R)$ is path connected, there is a sequence of paths $\gamma_{n}$ in $H\left(T_{1}(R)\right)$, with $\gamma_{n}(0)=x_{n}$ and $\gamma_{n}(1)=y_{n}$. By analytical continuation along $\gamma_{n}$, we can force the sequence $\left\{\gamma_{n}\right\}$ to converge uniformly to a path $\gamma$, in $T_{2}(R)$, connecting $x$ with $y$. Hence $T_{2}(R)$ is path connected.

Let $\phi \in \operatorname{Mod}_{2}(R)$, if $\phi\left(H\left(T_{1}(R)\right)\right) \cap H\left(T_{1}(R)\right) \neq \emptyset$, then $\phi \in \alpha\left(\operatorname{Mod}_{1}(R)\right)$, and $\phi\left(H\left(T_{1}(R)\right)\right)=H\left(T_{1}(R)\right)$. On the other hand, if $\phi \in \operatorname{Mod}_{2}(R) \backslash \alpha\left(\operatorname{Mod}_{1}(R)\right)$, then $\phi\left(H\left(T_{1}(R)\right)\right) \cap H\left(T_{1}(R)\right)=\emptyset$. This shows that $T_{2}(R)$ is not path connected if $\operatorname{Mod}_{2}(R) \backslash \alpha\left(\operatorname{Mod}_{1}(R)\right) \neq \emptyset$. In fact, $T_{2}(R)$ is decomposed into path connected components by $H\left(T_{1}(R)\right)$ and its orbit under the action of $\operatorname{Mod}_{2}(R) / \alpha\left(\operatorname{Mod}_{1}(R)\right)$.

Example 3.6. Let us consider the map $F(z)=z^{n}$, the Julia set is the unit circle $\mathbb{S}^{1}$. Let $\phi \in \operatorname{Mod}_{2}(F)$, by composing with a rotation, we can assume that $\phi(1)=1$. Any orientation preserving automorphism of the unit circle that fixes 1, and commutes with the dynamics of $F$, must be the identity. This is so, since such an automorphism must fix every point in the grand orbit of 1 , and every grand orbit is dense in $\mathbb{S}^{1}$. Thus, $\phi$ restricted to $\mathbb{S}^{1}$ is the identity. Taking a suitable homotopic representative of $\phi$, we can assume that $\phi$ leaves a tubular neighborhood of $S^{1}$ invariant. The dynamics on this tubular neighborhood have a fundamental domain homeomorphic to an annulus. Thus $\phi$ induces an oriented quasiconformal automorphism of this annulus. The group of oriented quasiconformal automorphisms of an annulus is generated by a Dehn twist of angle $2 \pi$.

Let $\tau$ be this generator. Since $\phi$ commutes with dynamics, $\tau$ must be propagated to the grand orbit of the fundamental annulus. A preimage of $\tau$ has the angle $2 \pi / n$. A forward image increases the angle by $2 \pi n$. But $\phi$ is defined on a neighborhood $U$ of $\mathbb{S}^{1}$. Then, $\tau$ only iterates finitely many times in $U$. Thus, the total angle is bounded, and then the map induced by $\tau$ in $U$ can be continuously deformed to the identity. This extends to every map generated by $\tau$.

By the assumption above, any element in $\operatorname{Mod}_{2}(F)$ is represented by a rotation which can be globally extended to an element in $\operatorname{Mod}_{1}(F)$. The homomorphism $\alpha$ is surjective; hence, by Theorem $3.5 T_{2}(F)$ is path connected. If $G$ is a hyperbolic 
Blashke map, then $G$ restricts to a degree $n$ expanding map on $\mathbb{S}^{1}$, so $G$ is locally conjugated to $F$. If $G$ is a Blashke map, then the map $\alpha$ is surjective and $T_{2}(G)$ is path connected.

The previous example motivates the following proposition.

Theorem 3.7. Let $P$ be a polynomial, then $T_{2}(P)$ is path connected if, and only if, the Julia set $J(P)$ is connected.

Proof. Assume that $J(P)$ is not connected, then there exist at least two disjoint Jordan curves $\gamma_{1}$ and $\gamma_{2}$, contained in the Fatou set, such that $P\left(\gamma_{1}\right)=P\left(\gamma_{2}\right)$, and the interior of each curve intersects a piece of the Julia set. We can take $\gamma_{1}$ and $\gamma_{2}$ such that, the image of these curves do not intersect the postcritical set. Let $\phi:=(\phi, U)$ be the element in $\operatorname{Mod}_{2}(P)$, defined by a Dehn twist on $\gamma_{1}$ and acting as the identity in $\gamma_{2}$. Using dynamics, extend these actions to the grand orbit of $\gamma_{1}$ and $\gamma_{2}$. Then, $\phi$ cannot be extended continuously to a global map in $\operatorname{Mod}_{1}(P)$, commuting with dynamics of $P$. This is because the action, of the extension of $\phi$, is homotopically different in two preimages of $P\left(\gamma_{1}\right)$.

Now, let us suppose that $J(P)$ is connected and let $\phi$ be an element in $\operatorname{Mod}_{2}(P)$. We will extend $\phi$ to a globally defined map in $\operatorname{Mod}_{1}(P)$. Since $P$ is a polynomial, $\infty$ is a superattracting fixed point. If $\operatorname{deg}(P)=d$, by Böttcher's Theorem, $P$ is conjugated to $z^{d}$ on the basin of attraction $A_{0}(\infty)$.

As we showed in Example 3.6, $\phi$ can be extended to $A_{0}(\infty)$ and, the action of $\phi$ on $A_{0}(\infty)$ is either a rotation or the identity. But $J(P)=\partial\left(A_{0}(\infty)\right)$, then the boundary of each Fatou component is either fixed by $\phi$ or, is moved to another component by a rotation. In either case, $\phi$ interchanges Fatou components univalently. Then, it is enough to extend the map on each periodic component. Once it is done, we use the dynamics of $P$ to extend to preperiodic components.

Let us check that we can extend $\phi$ to every periodic Fatou component $W$. There are three cases; if $W$ is hyperbolic, then $P$ is conjugated on $W$ to a hyperbolic Blashke map so, by Example 3.6, $\phi$ can be extended to $W$.

If $W$ is a Siegel disk, then $\phi$ is defined on $U$, a neighborhood of $J(P)$. We can modify $\phi$ using a homotopy, so that $\phi$ leaves invariant a rotational leaf $L$ of the Siegel foliation of $W$. Since $\phi$ is quasiconformal in $U$, the restriction of $\phi$ to $L$ is quasi-regular. Hence, we can radially extend $\phi$ to a quasiconformal map in $W$.

Finally, we discuss the case where $W$ is a parabolic Fatou component. Let $K=W \backslash U$ be the compact set where the map $\phi$ is not defined. The neighborhood $U$ contains a horodisk $D$, induced by the parabolic dynamics of $P$ in $W$. It also contains all the $P^{n}$-preimages of $K$, for a sufficiently large $n$. Thus $P^{n}$ has a lifting from $K$ to $U$. Let $C_{v}=\left\{v_{1}, v_{2}, \ldots, v_{m}\right\}$ be the set of critical values in $W$, and $*$ be a given point in $(U \cap W) \backslash C_{v}$. By Hurwitz' Theorem, the map $P^{n}$ induces an isomorphism of the fundamental group $\pi_{1}\left(W \backslash C_{v}, *\right)$.

Hence, given a point $x$ in $K$ such that $P(x) \in D$. Take $y \in P^{-n}(x)$, and define $\phi(x)=P^{n}(\phi(y))$. As a consequence of the Hurwitz argument above, $\phi(x)$ does not depend on the point $y$. Moreover, any homotopy that moves the point $y$, must move all other elements in $P^{-n}(x)$, since the map induced by $P^{n}$ in $\pi_{1}\left(W \backslash C_{v}, *\right)$ is an isomorphism. Also, $P^{k}$ and $\phi$ are defined in $U$ and commute for all $k \geq n$. Thus we have $\left.P(\phi(x))=P^{n+1}(\phi(y))\right)=\phi\left(P^{n+1}(y)\right)=\phi(P(x))$, so the extension of $\phi$ in $P^{-1}(D) \cap K$ commutes with $P$. The extension is quasiconformal since $P$ is holomorphic. Finally, using the dynamics of $P$, we extend $\phi$ to $K$. 


\section{Maps With totally Disconnected Julia SETS}

We now restrict the discussion to the case where the Julia set $J(R)$ is homeomorphic to a Cantor set. Under these conditions, we show that the Teichmüller space $T_{2}(R)$ has a product structure. Let us recall the proof of the following known fact.

Lemma 4.1. Let $P$ be a unimodal polynomial such that $J(P)$ is totally disconnected, then $\operatorname{Mod}_{1}(P)$ is generated by a single Dehn twist.

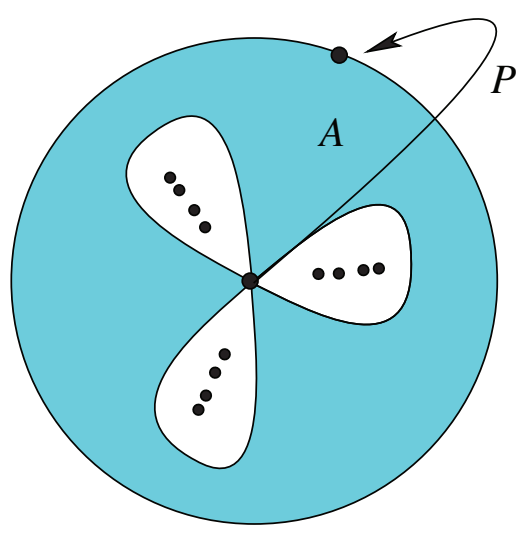

Figure 1. Critical annulus for Cantor dynamics.

Proof. Let $d$ be the degree of $P$. Consider a simple close path $\gamma$ through the critical value in the dynamical plane, and such that $\gamma$ contains the Julia set $J(P)$ in its interior. The preimage of $\gamma$ consist of $d$ closed loops, based on the critical point (see Figure 1). Let $A$ be the annulus defined by the intersection of the interior of $\gamma$ with the exterior of $P^{-1}(\gamma)$. Any global automorphism of $\mathbb{C}$, commuting with the dynamics of $P$, must leave the annulus $A$ invariant. Hence, the group of such automorphisms is generated by a Dehn twist defined on $A$.

Let $S$ be a multiply connected Riemann surface with boundary such that the connected components of $\partial S$ are Jordan curves. The pure mapping class group $\operatorname{Map}(S)$ is defined by the set of topological automorphisms of $S$, acting identically on the boundary, modulo a homotopic relation. This homotopic relation is defined as follows, $f \sim g$ are equivalent if, and only if, there exists an isotopy $H$, from $f$ to $g$, such that $\left.H\right|_{\partial S}=\left.f\right|_{\partial S}=\left.g\right|_{\partial S}$. A classical theorem states that the group $\operatorname{Map}(S)$ is generated by Dehn twists along simple closed curves.

Let $P$ be a unimodal polynomial of degree $d$, such that the Julia set $J(P)$ is homeomorphic to a Cantor set. This is equivalent to saying that the critical orbit of $P$ escapes to infinity. Let $\gamma$ be a Jordan curve, whose interior contains the Julia set and the critical value is outside $\gamma$. The preimage $P^{-1}(\gamma)$ consists of $d$ disjoint Jordan curves and, together with $\gamma$, defines a $d+1$-connected Riemann surface $S_{1}$ 
with boundary. Define recursively $S_{n}$ by $S_{n}=P^{-1}\left(S_{n-1}\right)$. Then $\operatorname{Map}\left(S_{n+1}\right)$ is the $d$-fold product of $\operatorname{Map}\left(S_{n}\right)$. We have the following:

Lemma 4.2. Let $P$ be a unimodal polynomial such that $J(P)$ is homeomorphic to a Cantor set, then $\operatorname{Map}\left(S_{n}\right)$ is embedded into $\operatorname{Mod}_{2}(P)$. Thus $\longrightarrow$ lim $\operatorname{Map}\left(S_{n}\right)$ is also embedded into $\operatorname{Mod}_{2}(P)$.

Proof. The embedding from $S_{n}$ to $S_{n+1}$, induces a monomorphism from the group $\operatorname{Map}\left(S_{n}\right)$ to the group $\operatorname{Map}\left(S_{n+1}\right)$. To conclude the lemma, we show that every element in $\operatorname{Map}\left(S_{n}\right)$ induces a non-trivial element in $\operatorname{Mod}_{2}(P)$. Let $\tau$ be a Dehn twist along a simple closed curve $\gamma$. Using dynamics of $P$, we propagate $\tau$ along the great orbit of $\gamma$. This defines an element in $\operatorname{Mod}_{2}(P)$. Thus, we have a map $\Phi_{n}: \operatorname{Map}\left(S_{n}\right) \rightarrow \operatorname{Mod}_{2}(P)$. If $\tau \neq \tau^{\prime}$ in $\operatorname{Map}\left(S_{n}\right)$, then $\tau$ and $\tau^{\prime}$ have different rotation numbers along the same curves. But, this property is preserved by the dynamics of $P$ and then $\Phi_{n}(\tau) \neq \Phi_{n}\left(\tau^{\prime}\right)$. So $\Phi_{n}$ is an injective map.

Note that if, instead of $\operatorname{Map}\left(S_{n}\right)$, we consider the group of automorphisms of $S_{n}$ not necessarily acting identically on $\partial S_{n}$. Then, on the corresponding product, it appears to be the action of a braiding group.

It is not clear that every element in $\operatorname{Mod}_{2}(R)$, acting identically on $J(R)$, should be homotopic to some element in $\operatorname{Map}\left(S_{n}\right)$. Moreover, $\operatorname{Mod}_{2}(R)$ consists of elements that have a simplicial extension; this relates the modular group $\operatorname{Mod}_{2}(R)$ with Thompson's group of automorphisms of the Cantor set.

In general, $T_{2}(R)$ is not path connected since $T_{2}(R)$ contains $H\left(T_{1}(R)\right)$ and the orbit of $H\left(T_{1}(R)\right)$ under the action of $\operatorname{Mod}_{2}(R)$. Locally, the orbit space is homeomorphic to

$$
\operatorname{Mod}_{2}(R) / \alpha\left(\operatorname{Mod}_{1}(R)\right) .
$$

Thus we have the following lemma.

Lemma 4.3. If the homomorphism $\alpha$ is not surjective, the space

$$
\operatorname{Mod}_{2}(R) / \alpha\left(\operatorname{Mod}_{1}(R)\right)
$$

is totally disconnected.

Proof. Assume that there is a path $\sigma:[0,1] \rightarrow \operatorname{Mod}_{2}(R) / \alpha\left(\operatorname{Mod}_{1}(R)\right)$. But, the path $\sigma$ induces a homotopy between $\sigma(0)$ and $\sigma(1)$, for all $t \in[0,1]$. Hence $\sigma$ is a constant map in $\operatorname{Mod}_{2}(R)$.

Theorem 4.4. Let $P$ be a hyperbolic unimodal polynomial such that $J(P)$ is homeomorphic to a Cantor set. Then

$$
T_{2}(P)=H\left(T_{1}(P)\right) \times\left\{\operatorname{Mod}_{2}(P) / \alpha\left(\operatorname{Mod}_{1}(P)\right)\right\} .
$$

Moreover, the space

$$
\operatorname{Mod}_{2}(P) / \alpha\left(\operatorname{Mod}_{1}(P)\right)
$$

is perfect.

Proof. Let us first check, that the space $\operatorname{Mod}_{2}(P) / \alpha\left(\operatorname{Mod}_{1}(P)\right)$ is perfect. By Lemma 4.2, $\operatorname{Mod}_{2}(P)$ contains $\lim \operatorname{Map}\left(S_{n}\right)$. Let $\gamma_{1}$ be a simple closed curve in $S_{n}$. For every $n>1$, choose a component $\gamma_{n}$ of $P^{-n}(\gamma)$. Let $g_{n}$ be the map, in $\operatorname{Mod}_{2}(P)$, induced by the Dehn twist of angle $2 \pi$ along $\gamma_{n}$ and acting as the identity around all other components of $P^{-n}(\gamma)$. 
Then, the maps $g_{n}$ are different, and by construction, $g_{n}$ cannot be extended to a globally defined element in $\operatorname{Mod}_{1}(P)$ commuting with dynamics. Moreover, the $g_{n}$ belong to different orbits of the action of $\alpha\left(\operatorname{Mod}_{1}(P)\right)$. Thus, the maps $g_{n}$ induce different elements in $\operatorname{Mod}_{2}(P) / \alpha\left(\operatorname{Mod}_{1}(P)\right)$. Now, the distortions satisfy $K\left(g_{n}\right)=$ $K\left(g_{1}\right)$, for all $n$. Hence, the map $g_{n}(I d)$ belongs to the ball $B\left(I d, K\left(g_{1}\right)+1\right)$ in $T_{2}(P)$. This implies that there exists an accumulation point in $B\left(I d, K\left(g_{1}\right)+1\right)$ and then there is an accumulation point in $\operatorname{Mod}_{2}(P) / \alpha\left(\operatorname{Mod}_{1}(P)\right)$. Since the action of the group $\operatorname{Mod}_{2}(P)$ is transitive in $\operatorname{Mod}_{2}(P) / \alpha\left(\operatorname{Mod}_{1}(P)\right)$, it follows that the fiber $\operatorname{Mod}_{2}(P) / \alpha\left(\operatorname{Mod}_{1}(P)\right)$ is perfect.

By Lemma 4.1, the map $\alpha$ is injective and then $H\left(T_{1}(P)\right) \simeq T_{1}(P)$ by Proposition 3.4. Since $\operatorname{Mod}_{1}(P)$ acts properly discontinuously on $T_{1}(P)$, there exists $r_{0}>0$ such that the ball $B\left(I d, r_{0}\right)$, in $T_{1}(P)$, projects injectively into $Q C(P)$. Then $B\left(I d, r_{0}\right)$ embeds injectively on $\operatorname{Hyp}(P)$, and the image of $B\left(I d, r_{0}\right)$ in $\operatorname{Hyp}(P)$ is evenly covered by the projection of $T_{2}(P)$ into Hyp $(P)$. Let $U$ be the open component, in the fiber of $B\left(I d, r_{0}\right)$, containing the identity in $T_{2}(P)$. By construction, $U \cap \operatorname{Mod}_{2}(P) / \alpha\left(\operatorname{Mod}_{1}(P)\right)=I d$. Then, $I d$ has a neighborhood in $T_{2}(P)$ of the form

$$
U \times \operatorname{Mod}_{2}(P) / \alpha\left(\operatorname{Mod}_{1}(P)\right) .
$$

The same argument works for every $x \in T_{2}(P)$. Hence $T_{2}(P)$ is homeomorphic to the product $T_{1}(P) \times \operatorname{Mod}_{2}(P) / \alpha\left(\operatorname{Mod}_{1}(P)\right)$.

Conceivably, $T_{2}(R)$ is also locally a product when $R$ is a rational map with disconnected Julia set. However, in this case, a non-trivial monodromy may appear along $H\left(T_{1}(R)\right)$.

Now we will see that the connectivity of $J(R)$ is related to the injectivity of $\alpha: \operatorname{Mod}_{1}(R) \rightarrow \operatorname{Mod}_{2}(R)$.

Theorem 4.5. Let $P$ be a hyperbolic polynomial. The map $\alpha: \operatorname{Mod}_{1}(P) \rightarrow$ $M_{o d}(P)$ is injective if, and only if, $P$ is unimodal and the Julia set $J(P)$ is homeomorphic to a Cantor set.

Proof. Let us assume that $P$ is unimodal, and $J(P)$ is homeomorphic to a Cantor set. By Lemma 4.1, the modular group $\operatorname{Mod}_{1}(P)$ is cyclically generated by a Dehn twist $\tau$, but $\tau$ is non-trivial in $\operatorname{Mod}_{2}(P)$. Hence, $\alpha$ is injective.

Reciprocally, by the hyperbolicity of $P$, the critical point in $\mathbb{C}$ is attracted to a periodic cycle in the plane. Without loss of generality, we can assume that this periodic cycle is a fixed point $z_{0}$. The immediate basin of attraction $A\left(z_{0}\right)$ is a topological disk. Let $A$ be an annulus inside $A\left(z_{0}\right)$, with center at $z_{0}$, such that $P$ maps the outer boundary of $A$ to the inner boundary of $A$. Consider a Dehn twist along the core curve of $A$ and propagate it along its grand orbit using dynamics. The resulting map $\tau$ is a non-trivial element of $\operatorname{Mod}_{1}(P)$. However, as we saw in Example 3.6. near the boundary of $A\left(z_{0}\right), \tau$ is homotopic to the identity. Thus, $\tau$ is the identity in $\operatorname{Mod}_{2}(P)$ and $\alpha$ is not injective in $\operatorname{Mod}_{1}(P)$. Then, the critical point is attracted to infinity. Hence, the Julia set $J(P)$ is homeomorphic to a Cantor set.

Example 4.6. A useful example is $f_{10}(z)=z^{2}+10$. In this case, the Julia set is a Cantor set. So the group $\operatorname{Mod}_{1}\left(f_{10}\right)$ is cyclically generated by a Dehn twist. It follows that $T_{1}\left(f_{10}\right)$ is homeomorphic to the puncture unit disk. The quotient space is equivalent to the complement of the Mandelbrot set $M$. It is well known, 
that $\mathbb{C} \backslash M$ is holomorphically equivalent to the puncture unit disk. Also, since $\operatorname{Mod}_{2}\left(f_{10}\right)$ is infinitely generated, the homomorphism $\alpha$ is not surjective.

\section{INVERSE LIMITS OF RATIONAL FUNCTIONS AND ITS DEFORMATIONS}

Let us consider a rational map $R: \overline{\mathbb{C}} \rightarrow \overline{\mathbb{C}}$ acting on the Riemann sphere. The inverse limit, or natural extension of $R$, is the space

$$
\mathcal{N}_{R}=\left\{\hat{z}=\left(z_{1}, z_{2}, \ldots\right) \in \prod_{n \in \mathbb{N}} \overline{\mathbb{C}}: R\left(z_{n+1}\right)=z_{n}\right\}
$$

endowed by the Tychonoff topology as a subspace of $\prod_{n \in \mathbb{N}} \overline{\mathbb{C}}$. There is a family of natural projections $p_{n}: \mathcal{N}_{R} \rightarrow \overline{\mathbb{C}}$ given by $p_{n}(\hat{z})=z_{n}$, also a natural extension of $R$, denoted by $\hat{R}: \mathcal{N}_{R} \rightarrow \mathcal{N}_{R}$ such that $p_{n} \circ \hat{R}=R \circ p_{n}$. To simplify notation, let us put $p:=p_{1}$.

The space $\mathcal{N}_{R}$ was studied by Lyubich and Minsky in [2]. In that paper, Lyubich and Minsky showed that for a general rational map, the natural extension is decomposed into two spaces: the regular part $\mathcal{R}_{R}$, which consists of the points that admit a Riemannian structure compatible with the maps $p_{n}$ and the complement of $\mathcal{R}_{R}$ called the irregular part. A leaf $L$ is a path-connected component in $\mathcal{R}_{R}$. Every leaf is a Riemann surface. A theorem by Lyubich and Minsky shows that, in $\mathcal{R}_{R}$, there is a family of leaves, such that, each leaf in this family is conformally equivalent to the plane and it is dense in $\mathcal{N}_{R}$.

The authors of 2, proved that there is a class of rational maps $R$, which contains all hyperbolic maps, such that $\mathcal{R}_{R}$ is a lamination by Riemann surfaces. That is, it admits an atlas of charts $(U, \phi)$, where $\phi$ is a homeomorphism from $U$ to $\mathbb{D} \times T$. Changes of coordinates are conformal on the horizontal direction and continuous in the transversal direction. This structure is consistent with the fibration induced by the family of maps $p_{n}$.

Let $P(R)$ denote the postcritical set of $R$. If $z_{0}$ is a given point in $\mathbb{C} \backslash P(R)$, then a construction due to Poincaré gives a representation of the fundamental group $\pi_{1}\left(\mathbb{C} \backslash P(R), z_{0}\right)$ into the automorphisms group of the fiber $p^{-1}\left(z_{0}\right)$. The image of this representation is called the monodromy group of $\mathcal{N}_{R}$. Because of the irregular part, the natural extension is not the suspension of $\mathbb{C}$ by the monodromy group on the fiber $p^{-1}\left(z_{0}\right)$.

5.1. Deformations of inverse limits. Consider an open neighborhood $U$ of the Julia set $J(R)$; we call the fiber $p^{-1}(U)$ a maximal flow box for $\mathcal{N}_{R}$. The action of the monodromy group induces identifications on a maximal flow box. We say that $\mathcal{N}_{R}$ is represented by a maximal flow box $F$ with the action of monodromy, if $\mathcal{N}_{R}$ coincides with the end compactification of the orbit by the action of this monodromy on $F$. A maximal flow box contains almost all irregular points, so we can always represent $\mathcal{N}_{R}$ by a maximal flow box with the action of monodromy. A maximal flow box contains almost all irregular points, so we can always represent $\mathcal{N}_{R}$ by a maximal flow box and the action of monodromy. When $R$ is hyperbolic, the regular part is a Riemann surface lamination and the irregular part is finite; see 2 .

Then any conjugacy, around a neighborhood of the Julia set of $R$, can be extended to a homeomorphism of the whole laminations. This suggests that we can extend the equivalence class of elements in $T_{2}(R)$ to equivalence classes of laminations. In this sense, the monodromy and the dynamics characterize laminations. 
Then, deformations of the whole lamination are determined by deformations of a maximal flow box.

Let $U$ be a neighborhood in $\mathcal{N}_{R}$; we call a plaque a path component of $U \cap \mathcal{N}_{R}$. A map $\gamma$, continuously defined on plaques or open neighborhoods in $\mathcal{N}_{R}$, is called a fiber automorphism if $p \circ \gamma=p$. Since $p$ is holomorphic in the regular part, it implies that $\gamma$ is holomorphic in $\mathcal{R}_{R}$. Given a fiber automorphism and a leaf $L$ in $\mathcal{R}_{R}$, we denote by $\gamma_{L}$ the restriction of $\gamma$ to $L$ whenever it is defined.

Definition 5.1. Let $U$ be a neighborhood in $\mathbb{C}$ and $F=p^{-1}(U)$ a flow box. A family $\left\{\mu_{L}\right\}$ of Beltrami differentials, defined on $F$, is called compatible with the fiber structure if, for every fiber automorphism $\gamma$ and every leaf $L$ in $\mathcal{R}_{R}$, we have on $F \cap \gamma(F) \cap L$,

$$
\mu_{\tilde{L}}\left(\gamma_{L}\right) \frac{\bar{\gamma}_{L}^{\prime}}{\gamma_{L}^{\prime}}=\mu_{\tilde{L}},
$$

where $\gamma_{L}$ sends $L$ into $\tilde{L}$.

Let $\left\{R^{-n}\right\}$ be the family of branches of $R$; then, deck transformations of the family of branches are fiber automorphisms. Moreover, all fiber automorphisms are generated by deck transformations of branches of $R$.

Lemma 5.2. Let $\mu=\left\{\mu_{L}\right\}$ be a family of Beltrami differentials in $\mathcal{R}_{R}$; then, $\left\{\mu_{L}\right\}$ is compatible with the fiber structure if, and only if, $p_{*} \circ p^{*}(\mu)=\mu$.

Proof. Assume that $\mu$ is compatible with the fiber structure, then it is invariant under all deck transformation of branches of $R$. Thus the pushforward $p^{*}\left(\mu_{L}\right)$ is independent of the leaf $L$ and, the pullback $p_{*} \circ p^{*}(L)$ is the same for all leaves $L$ and coincides with $\mu$.

The equation $p_{*} \circ p^{*}(\mu)=\mu$ implies that, the family $\mu$ is invariant under deck transformations of $R$. Hence $\mu$ must be compatible with the fiber structure.

Since the natural extension $\mathcal{N}_{R}$ is a metric space, we will consider quasiconformal maps, in Pesin's sense, defined on subsets of $\mathcal{N}_{R}$. Let $F$ be a maximal flow box for $\mathcal{N}_{R}$ and let $X\left(\mathcal{N}_{R}, F\right)$ be the space of surjective homeomorphisms $\phi: \mathcal{N}_{R} \rightarrow \mathcal{N}_{R_{1}}$, quasiconformal in Pesin's sense, which on $F$ conjugates the respective monodromy actions. This condition implies that $\phi$ induces a family of Beltrami differentials on $F$ that is compatible with the fiber structure.

We say that two maps $\phi: \mathcal{N}_{R} \rightarrow \mathcal{N}_{R_{1}}$ and $\psi: \mathcal{N}_{R} \rightarrow \mathcal{N}_{R_{2}}$, in $X\left(\mathcal{N}_{R}, F\right)$, are equivalent if there exists a map $\sigma: \mathcal{N}_{R_{1}} \rightarrow \mathcal{N}_{R_{2}}$, conformal in Pesin's sense, such that $\psi=\sigma \circ \phi$ and $\sigma$ is homotopic to the identity, with homotopy that commutes with dynamics and monodromy actions.

We define the space of deformations of $\mathcal{N}_{R}$, and denote it by $\operatorname{Def}\left(\mathcal{N}_{R}, F\right)$, as the set $X\left(\mathcal{N}_{R}, F\right)$ modulo the equivalence relation above. Since we are considering surjective homeomorphisms $\phi: \mathcal{N}_{R} \rightarrow \mathcal{N}_{R_{1}}$, it follows that $R$ and $R_{1}$ have the same degree.

Note that the image of $F$ under any map in $\operatorname{Def}\left(\mathcal{N}_{R}, F\right)$ is a maximal flow box of some rational map. We have the following:

Theorem 5.3. Let $R$ be a map that is represented by a maximal flow box. Then, there is a bijection between $\operatorname{Def}\left(\mathcal{N}_{R}, F\right)$ and the space $T_{2}(R)$.

Proof. Let $(h, U)$ be an element in $T_{2}(R)$, then $h$ induces a Beltrami differential $\nu$ around a neighborhood $U$ of $J(R)$. We consider the family of Beltrami differentials 
$\mu$ on a neighborhood of $p^{-1}(J(R))$. Since $R$ is represented by a maximal flow box, we can use dynamics and monodromy to propagate $\mu$ on all the inverse limits. By construction $p_{*} \circ p^{*}(\mu)=\mu$, so by Lemma 5.2, the resulting family of Beltrami differentials is compatible with the fiber structure. Thus $\mu$ induces an element in $\operatorname{Def}\left(\mathcal{N}_{R}, F\right)$ and, the construction only depends on the class of $(h, U)$ in $T_{2}(R)$.

Now, let $\phi$ be a representative of a point in $\operatorname{Def}\left(\mathcal{N}_{R}, F\right)$. Since $\phi$ conjugates dynamics and the monodromy actions, we can push $\phi$, using $p$, to get a quasiconformal map $h: U \rightarrow \mathbb{C}$, conjugating the corresponding rational maps. Then, $(h, U)$ defines an element in $T_{2}(R)$.

\section{ACKNOWLEDGMent}

We thank T. Kawahira for useful suggestions regarding the manuscript of this paper.

\section{REFERENCES}

1. X. Buff and A. L. Epstein, From local to global analytic conjugacies, Ergod. Th. \& Dynam. Sys. 27 (2007), no. 4, 1073-1094. MR2342966 (2008g:37039)

2. M. Lyubich and Y. Minsky, Laminations in holomorphic dynamics, J. Diff. Geom. 47 (1997), 17-94. MR 1601430 (98k:58191)

3. P. Makienko, Automorphisms of a rational function with disconnected Julia set, Orsay, Preprint, 031992.

4. R. Mañé, P. Sad, and D. Sullivan, On the dynamics of rational maps, Ann. Scien. Ec. Norm. Sup., Paris(4) (1983).

5. C. McMullen, Automorphisms of rational functions, Holomorphic functions and moduli I. (D. Drasin et al., eds.), Springer-Verlag, 1986, pp. 31-60. MR955807 (89m:58187)

6. C. McMullen and D. Sullivan, Quasiconformal homeomorphisms and dynamics III: The Teichmüller space of a holomorphic dynamical system, 1998.

7. D. Sullivan, Seminar on conformal and hyperbolic geometry by D. P. Sullivan (Notes by M. Baker and J. Seade), Preprint IHES, 1982.

Instituto de Matematicas UnAM, Av Universidad S/N Col lomas de Chamilpa CuerNavaCA, 62100 Cuernavaca MO, Mexico

E-mail address: carlos@matcuer.unam.mx

University Nacional Autonoma de Mexico, Institute of Mathematics, Av Universidad S/N, O P 62210 Morelos, Mexico

E-mail address: makienko@matcuer.unam.mx 